divine names, especially in theophoric personal names. ${ }^{44}$ Association with the storm god at Ugarit does not appear to derive from the east, but description of Baal as "the son of Dagan" might reflect inland Syrian use of the title Balu for Dagan. When the Emar scribes write their city god's name as ${ }^{\mathrm{d}}$ NIN.URTA, they make him Dagan's son by relation to Enlil, and the substitution of ${ }^{d}$ NIN.KALAM in one case might reveal an old Baal-title beneath the Sumerian ${ }^{d}$ NIN.URTA. When ${ }^{d}$ NIN.URTA is found with Dagan's wife Išhara, it may be neither adultery nor incest but reappearance of the father Dagan himself, in the oedipal guise of a son, through a title that takes on an independent existence.

\section{Zur Grammatik und zum Lexikon des Hurritischen*}

\section{von Gernot Wilhelm - Würzburg}

Zum Andenken an Johannes Friedrich anläßlich seines 100. Geburtstags am 27. August 1993

In Auseinandersetzung mit einer neueren Veröffentlichung von M. Dietrich und W. Mayer, die zahlreiche Ergebnisse der hurritologischen Forschung der letzten beiden Jahrzehnte in Frage stellt und in Grammatik und Lexikographie eigene Wege beschreitet, werden vor allem die folgenden Themen behandelt: Der Infinitiv auf -ummeni dessen Ansatz zuätzlich begriindet wird und desen Endung - Mi auf Trund der huitis Grund der huits de deren Interpretation als Absolutiv mit "Artikel“ -ne in Frage gestellt wird, da der Absolutiv anscheinend mit dem „Artikel“ nicht kompatibel ist.

Weiterhin diskutiert der Aufsatz verschiedene grammatische und lexikalische Deutungen der genannten Autoren und kommt durchgehend zu einem ablehnenden Urteil. Dabei werden insbesondere auch Fragen der Methodik angesprochen.

Die Erschließung der hurritischen Grammatik stützte sich im ersten halben Jahrhundert hurritologischer Forschung vornehmlich auf den Mittani-Brief ${ }^{1}$, dessen fast 500 Zeilen ganz oder großenteils erhaltenen Kontexts es gestatteten, zahlreiche Grammeme $\mathrm{zu}$ isolieren und in vielen Fällen ihre Funktion zu bestimmen, einen Grundstock von mehr oder minder exakt defi-

* Besondere Abkürzungen: AdŠ $=$ G. Wilhelm, Das Archiv des Šilwa-teššup (1980 ff.). $-\mathrm{ChS}=$ (ed.) V. Haas et al., Corpus der hurritischen Sprachdenkmäler (1984 ff.). - ChuJ = M. L. Chačikjan, Churritskij i urartskij jazyki (= Churrity i Urarty 2,1985$)$. $-\mathrm{EN}=$ Exeavations at Nuzi $(9 / 1)$, in: SCCNH 2 (1987) $355 \mathrm{ff}$ - $\mathrm{ERL}=\mathrm{E}$. R. Lacheman (Tontafelsignatur im Harvard Semitic Museum). - GHL $=\mathrm{F} \cdot \mathrm{W}$. Bush, A Grammar of the Hurrian Language (1964) - HuU = E. A. Speiser, Introduction to Hurrian (=AASOR 20, 1941). - SCCNH = Studies on the Civilization and Culture of Nuzi and the Hurrians (1981 ff.).

${ }^{1}$ Letzte Autographie: O. Schroeder, Die Tontafeln von El-Amarna, 2. Teil (VS 12, 1915) Nr. 200. Letzte Transliteration: J. Friedrich, Kleinasiatische Sprachdenkmäler (1932) 8-32; letzte Gesamtübersetzung: G. Wilhelm apud

W. L. Moran, The Amarna Letters (1992) 63-7I.

Zeitschr. f. Assyriologie Bd. 83, S. 99-118

C) Walter de Gruyter 1993

ISSN 0084-5299 
nierten Lexemen zu gewinnen, die wesentlichen Muster sowohl der Morpho- wie der Satzsyntax zu beschreiben und sogar Rückschlüsse auf die Phonemik und Phonetik zu ziehen, die über die bloße Übertragung der phonematischen Information akkadischer Silbenzeichen hinausgingen. Die dabei gewonnenen Ergebnisse fanden 1941 ihren Niederschlag in der Grammatik von E. A. Speiser ${ }^{2}$, die in der 1964 vorgelegten Dissertation von F. W. Bush durch wichtige Präzisierungen und Korrekturen ergänzt wurde ${ }^{3}$. Die neuere Forschung zur hurritischen Grammatik hat insbesondere Impulse durch den hurritisch-urartäischen Sprachvergleich erfahren, der in der vergleichenden Grammatik I. M. Diakonoffs von $1971^{4}$ - überarbeitet und ergänzt in einer russischen Ausgabe von M. L. Chačikjan von $1985^{5}$ - eine erste systematische Gesamtdarstellung gefunden hat. Ein anderer wichtiger Impuls ging von der Erschließung hurritischer Quellen anderer Fundorte aus, wobei sich zunächst die mehrsprachigen Vokabulare sowie die akkadisch-hurritische Bilingue aus Ugarit, dann auch infolge der Gewinnung größerer, zusammenhängender Textstücke die hurritischen Beschwörungen, Gebete und Mythen aus Boğazköy als besonders ergiebig erwiesen. Eine gänzlich neue Situation ergab sich 1983 durch den glücklichen Fund einer hurri tisch-hethitischen Bilingue ${ }^{6}$, die derzeit im Mittelpunkt der hurritologischen Diskussion steht. Daneben hat aber auch die Beschäftigung mit dem Mittani-Brief noch manche Detailerkenntnisse lexikalischer und morphologischer Art erbracht. Gemessen an der Bedeutung der Hurriter und des Hurritischen im altorientalischen Kulturzusammenhang ist die Zahl der einschlägig arbeitenden Altorientalisten immer noch außerordentlich gering, und so wird man es nur begrüßen können, wenn sich weitere Forscher diesem Felde zuwenden. Daß jede weitergehende Forschung von dem inzwischen erreichten Kenntnis- und Diskussionsstand ausgehen

\footnotetext{
2 Speiser, IH.

3 Bush, GHL.

${ }^{4}$ Diakonoff, $\mathrm{HuU}$.

5 Chačikjan, ChUJ.

${ }^{6}$ KBo. 32,10-104, 208-210, 212-214, 216. Einen ersten Gesamtüberblick gaben H. Otten, JbAWG 1984, 50-60, und E. Neu: Das Hurritische: Eine altorientalische Sprache in neuem Licht (AbhMainz 1988/3, 1988) (im folgenden: Neu, Das Hurritische); für eine analytische Bibliographie der Forschung von 1984 bi 1990 ef. G. Wilhelm, Or. 61 (1992) 122-141.
}

und die bewährten philologisch-sprachwissenschaftlichen Methoden berücksichtigen muß, bedarf keiner weiteren Begründung.

In einem längeren Aufsatz, mit dem sie erstmals das Gebiet der Grammatik des Mittani-Briefes betreten, haben M. Dietrich und W. Mayer die Ergebnisse hurritologischer Forschung der letzten zwei Jahrzehnte generell in Frage gestellt und in mehreren wichtigen Punkten sowie in zahlreichen Einzelheiten Gegenpositionen bezogen ${ }^{7}$. Thre Kritik nimmt ihren Ausgang bei der Behauptung, die betreffenden Autoren - namentlich werden (jedoch nur „beispielsweise“!) W. Farber, Ch. Girbal, F. Plank, M. Salvini und G. Wilhelm genannt - hätten die grundlegende Grammatik Diakonoffs, HuU, „nicht oder nur unzulänglich genutzt“ (S. 108). Unverständlicherweise trifft das Verdikt von Dietrich und Mayer, HuU „nicht oder nur unzulänglich“ genutzt zu haben, auch einen Aufsatz von W. Farber ${ }^{8}$, welcher bereits Anfang 1971 publiziert wurde, d.h. in demselben Jahr, in dem auch $\mathrm{HuU}$ erschienen ist. Auch zitieren sie in diesem Zusammenhang einen Aufsatz des Verfs. ${ }^{9}$, der gar nicht von „hurritologischen Fragen" (so S. 108, 1. Zeile) handelt, sondern erstmals die Suffixaufnahme beim Genetivattribut im Urartäischen nachweist, eine Erkenntnis, die inzwischen Gemeingut geworden ist ${ }^{10}$.

Der Vergleich der Grammatik Diakonoffs mit dem GAG W. von Sodens (S. 107) wird der Forscherleistung Diakonoffs in schöner Weise gerecht; gleichwohl liegt dem Vergleich der beiden Grammatiken ein Mißverständnis der hurritologischen Forschungssituation zugrunde. Der Wunsch nach einer hurritischen Grammatik, die alle wesentlichen grammatischen Phänomene der vorliegenden Texte

${ }^{7}$ M. Dietrich/W. Mayer, Beiträge zum Hurritischen (I), UF 23 (1991 [erschienen Herbst 1992]) 107-126; ein „Teil II“ ist für UF 24 angekündigt.

${ }^{8}$ W. Farber, Zu einigen Enklitika im Hurrischen (Pronomen, Kopula, syntaktische Partikeln), Or. 40 (1971) 29-66. Gegenüber der Pauschalkritik von Dietrich und Mayer ist festzuhalten, daß dieser Aufsatz zu den wichtigsten Arbeiten zur hurritischen Grammatik zwischen dem Frscheinen von GHL und dem von als Erg. mit Pron. Sg. 3., Bestimmung der Kurzformen der enklitischen Personalpronomina) sind inzwischen Gemeingut der Hurritologie geworden und haben auch Eingang in Chačikjan, ChUJ (S. 73, Anm. 4) gefunden.

${ }^{9}$ G. Wilhelm, Zur urartäischen Nominalflexion, ZA 66 (1976) 105-119.

${ }_{10}$ Cf. M. Salvini, RHA 36 (1978) 159 ff.; ders., AISN 1 (1979) 97-115; I. M. Diakonoff/S. M. Kashkai, RGTC 9 (1981) XII f.; M. van Loon, in: K. R. Veenhof, Schrijvend verleden (1983) 34 Anm. 3. 
beschreibt, wie GAG dies für das Akkadische leistete, wird noch lange unerfüllt bleiben, und eine Auffassung, die $\mathrm{HuU}$ in einer forschungshemmenden Weise kanonisiert, kann sich am allerwenigsten auf Diakonoff selbst berufen, der jederzeit bereit war und ist, wohlbegründete neue Ergebnisse auch dann zu akzeptieren, wenn sie von seinem 1971 formulierten Erkenntnisstand abweichen. Dies wird z.B. daran deutlich, daß Diakonoff sich der Mühe unterzogen hat, drei Aufsätze des Verfs. - darunter zwei der von Dietrich und Mayer pauschal kritisierten - selbst ins Russische zu übersetzen und für ihre Veröffentlichung zu sorgen ${ }^{11}$.

Leider sind die Beiträge von Dietrich und Mayer nicht geeignet, die Forschung bei ihrem Bemühen um ein besseres Verständnis der Grammatik und des Lexikons des Hurritischen zu fördern, wie in der Diskussion einiger Thesen der beiden Autoren deutlich werden soll. Wenn hier bei weitem nicht jeder Punkt angesprochen werden kann, ist dies keinesfalls als Zustimmung des Verfs. zu werten

\subsection{Der Infinitiv auf -ummene/i}

Um die Vorgehensweise von Dietrich und Mayer zu verdeutlichen, sei zunächst der letzte Abschnitt ihres Aufsatzes erörtert, bei dem es sich teilweise um eine akkadistische Problematik handelt.

1987 hat Verf. eine Konstruktion mit einem bis dahin nicht beobachteten Infinitiv auf -ummeni in den hurro-akkadischen Urkunden aus Nuzi folgendermaßen beschrieben:

„Die ... Belege für die Infinitivendung -ummeni zeigen gegenüber den viel zahlreicheren für die Endung -umma eine syntaktische Gemeinsamkeit: In allen Fällen ist festzustellen, daß dem hurritischen Infinitiv die Präposition ana vorausgeht. Der Infinitiv ist hier nicht Teil des Prädikats wie stets in den Konstruktionen des Typs -umma epēšu, sondern eine adverbiale Bestinm den des Zwecks. Die beiden Konstruktionen ana hawum $(m)$ ini niqtabi unung kum [mmen]i a i bi-mi kuru $[m m e n] i \ldots i q b i-m i$ entsprechen genau rein akkadischen Konstruktionen

Dietrich und Mayer behaupten nun (S. 125), an allen fünf vom Verf. gesammelten und für einen Infinitiv auf -ummene/ $i$ in Anspruch genommenen Stellen habe er das Zeichen DÙ als NI

${ }^{11}$ G. Vil'gel'm, K churritskoj i urartskoj grammatike, Drevnij Vostok 5 (1988) 93132.

12 Wilhelm, Zum hurritischen Infinitiv in Nuzi, in: SCCNH 2 (1987) 336. verlesen“. Die beiden Autoren verschweigen dabei, daß an allen Stellen - soweit voll erhalten - nach den vorliegenden Autographien (in drei Fällen auch nach Kollation) der jeweilige Text das Zeichen NI, nicht DÙ bietet ${ }^{13}$. Von einer „Verlesung“ kann also keine Rede sein, vielmehr nehmen Dietrich und Mayer in allen Fällen eine Emendation von NI zu DÙ vor, ohne dies deutlich zum Ausdruck zu bringen und es in ihren Umschriften durch ein Ausrufungszeichen $\mathrm{zu}$ vermerken, wie es philologischer Korrektheit entsprochen hätte.

Der Versuch einer Rechtfertigung für dieses Vorgehen ist implizit in ihrer weiteren Behauptung enthalten, „die NuziKursive“ mache „Zwischen diesen beiden Zeichen keine markanten Unterschiede" (S. 125). Einen Beweis für diese Behauptung bleiben die beiden Autoren schuldig. Demgegenüber wird schon bei oberflächlicher Prüfung des Sachverhalts klar, daß in den NuziTexten das Zeichen DÙ in aller Regel mit nur einem senkrechten $\mathrm{Keil}^{14}$, das Zeichen NI mit zwei senkrechten Keilen geschrieben wird. Davon abweichende Fälle sind so selten, daß man sie als Fehler bezeichnen muß und also emendieren darf $^{15}$. Es erstaunt, daß dieser jedem Nuzi-Kenner wohlvertraute und gänzlich unzweifelhafte, dazu leicht nachprüfbare Sachverhalt dem Mitautor Mayer, der sich nach Ausweis seiner Habilitationsschrift ${ }^{16}$ längere Zeit intensiv mit den Nuzi-Texten beschäftigt hat, unbekannt geblieben sein sollte. Da man gleichwohl nicht annehmen möchte, hier werde gegen besseres Wissen argumentiert, muß der Vorwurf einer bedenklichen Leichtfertigkeit erhoben werden.

Nachdem Dietrich und Mayer sich auf diese Weise die Lizenz genommen haben, NI als DÙ zu lesen, schlagen sie vor, ana . . . DÙ als finalen Infinitiv zu verstehen, zu dem der hurritische Infinitiv das direkte Objekt sei und als solches der Kasusattraktion unterliege (S. 125 Anm. 63: „ana gewarumme epēše“). Der Auslaut der

${ }_{13}$ TCL 9,12+:21, letztes Zeichen; HSS 15,193:4, vorletztes Zeichen; AdŠ $84=$ HSS 14,606:15, letztes Zeichen; JEN 370:5, letztes Zeichen.

${ }_{14}$ Cf. JEN $1: 3,5: 4,9: 4,10: 4,35: 4,36: 4,43: 4,58: 4,66: 4$ a , $73: 6,74: 4,75: 4$, $81: 4,96: 4,97: 6,211: 3,734: 3,770: 4,774: 4$; HSS $5,66: 6,104: 18 ; 19,7: 43$, $37: 4,38: 10,62: 27$, EN IX/1 $2: 4,43: 6,48: 3$, etc.

${ }_{15}$ In dem Anm. 12 genannten Aufsatz, S. 336 Anm. 35, wird ein Beispiel für diesen Fall gegeben. Unter den ca. 100 Belegen für Dù in den (unvollständigen) Würzburger Nuzi-Sammlungen finden sich noch drei weitere solche Fälle: JEN $330: 14,435: 31$, HSS $5,67: 37$.

${ }^{16}$ Mayer, Nuzi-Studien I (AOAT 205/1, 1978). 
Endung -umme ist also den beiden Autoren zufolge nicht hurritisch zu erklären - im Mit.-Brief, in Ugarit und in Boğazköy lautet die Infinitivendung bekanntlich -umme -, vielmehr handelt es sich ihrer Meinung nach um die akkadische (genauer: die assyrische) Genetivendung.

Dietrich und Mayer beachten dabei jedoch nicht, daß Dù in Nuzi ganz überwiegend mit phonetischem Komplement, Pronominalsuffix, Enklitikon oder Pluraldeterminativ geschrieben wird ${ }^{17}$. Es wäre also sehr ungewöhnlich, wenn gerade bei der fraglichen Konstruktion stets das bloße Logogramm Dù stünde. Dietrich und Mayer beachten weiterhin nicht, daß der Infinitiv von epēšu in Nuzi

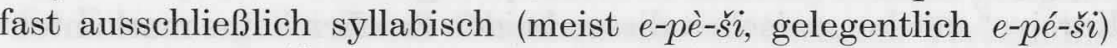
geschrieben wird ${ }^{18}$. In einem fast 2000 Texte umfassenden (Teil-) Corpus steht neben 41 Belegen für die vollsyllabische Schreibung ein einziger Beleg für die logographische Schreibung des Infinitivs von epéšu; jedoch weist dieser immerhin ein phonetisches Komplement auf ${ }^{19}$. Dietrich und Mayer versäumen es, ihre Interpretation durch den Nachweis von Schreibungen des Infinitivs mit dem bloßen Logogramm DÙ zu stützen, was allerdings wohl auch schwerlich möglich ist. Ferner bleiben sie eine Erklärung schuldig, warum gerade bei den vom Verf. gesammelten hurritischen Infinitiven der Genetiv regelmäßig als - e erscheinen soll, während er in Nuzi sonst regelmäßig $-i$ lautet $^{20}$. Schließlich unterlassen die beiden Autoren es, ihre Umdeutung von ge-wa-mu-um-me-né-e HSS 15, 193:4 als "ge-wa-ru-um-me DÙ-e" (S. 126) durch weitere Belege einer solchen für Nuzi ganz ungewöhnlichen Komplementierung sowie durch syllabische Schreibungen des Genetivs des Infinitivs von epēšu mit $e$-Auslaut zu stützen.

Ein sechster Beleg für den Infinitiv auf -ummeni liegt in AdŠ 278 (=ERL 74) vor: [... síG] ana nah [la]pta ana ku-[ru?-u]m?-me-ni na-

17 Aus einem (Teil-)Corpus von ca. 2000 Texten kann Verf. neben ca. hundert Belegen für DÙ mit -ma, -sú, -sú-ma, -šu, -šu-ma, -šu-mi, -š/šú, -šá, -mi, MEŠ nur drei oder vier für DÙ ohne phonetische Ergänzung oder Pluraldeterminativ nachweisen (JEN 10:4, 428:9, HSS 5,67:37 [oder mit Komplement?], 104:18). Diese Relation dürfte die Situation im gesamten Corpus widerspiegeln.

18 HSS $9,12: 4,39: 4,40: 3 ; 13,70: 10,103: 5,192: 4,193$ passim (7mal), 206:4, $449: 7 ; 14,540: 3,5,7$ (koll.); 15,192:2, 5, 8, $206: 2,6,10,210: 4,216: 5,218: 3$, $220: 5,17,24,36,299: 3,300: 5,323: 4,335$ I 3; e-pé-ši HSS 13,20:3; 14,223:3, $15: 157: 4,223: 13$; AASOR 16,1:19, 20

19 -na 1-en sí-a-na-ti DÙ-š $i$ HSS $13,342: 1$.

${ }^{20}$ Für einige der insgesamt seltenen Formen auf - $e$ cf. Wilhelm, AOAT 9 (1970) 39. $d[i n]$ „[. . W Wolle] ist für das Herstellen(?) eines Mantels gegeben.“ Auch an dieser Stelle steht unzweifelhaft NI, nicht DÙ.

Mit der öfter bezeugten Form ši-um-me-ni, ši- $(i-) u / u$-um-mi-ni, $\check{s} i-i-u-u-u m-m i-n i^{21}$ liegt wahrscheinlich der Infinitiv auf -ummene/ $i$ auch in rein hurritischem Kontext vor.

Der Versuch, die Existenz des hurritischen Infinitivs auf -umme$n e / i \mathrm{zu}$ bestreiten, basiert also nicht nur auf einer unzulässigen Emendation sämtlicher einschlägigen Textstellen; er steht vielmehr auch im Widerspruch zu mehreren philologischen Gegebenheiten der Nuzi-Texte und darf daher als gescheitert betrachtet werden.

Die Bildungsweise dieses Infinitivs kann aber heute genauer bestimmt werden, als dies $1984^{22}$ möglich war. In dem genannten Aufsatz hatte Verf. formuliert (S. $337 \mathrm{f}$.):

„Die Dimension dieser Kasusendung (d. h. -ne/i, Verf.) ist im Mit.-Brief und im Boğazköy-Hurritischen als Ablativ und Instrumentalis bestimmt worden, was in völliger Harmonie mit dem Befund im Urartäischen steht, aber dem Kontext der hier behandelten Stellen nicht gerecht wird."

Inzwischen hat die 1983 in Boğazköy entdeckte hurritisch-hethitische Bilingue ergeben, daß der Kasus auf -ne/ $i$ (zur Problematik s. unten) auch als „Ortskasus, vornehmlich zur Angabe einer Rich tung auf die Frage 'Wohin?' "23 begegnet. Diese direktivische Funktion des $n e$-Kasus erklärt nun auch die Anwesenheit der Kasusendung $-n e / i$ in den hurritischen Infinitiven auf -ummeni nach akkad. ana.

\subsection{Der Kasus auf -ne/}

Die soeben angesprochene Kasusendung -ne/i nimmt in dem Aufsatz von Dietrich und Mayer breiten Raum ein. Die beiden Autoren beziehen sich auf einen Aufsatz des Verfs. von 1983, in dem Substantive des Mit.-Briefes und der hurr. Boğazköy-Texte mit der Endung -ne/i gesammelt und nach dem jeweiligen Kontext teils als Ablativ, teils als Instrumentalis bestimmt wurden ${ }^{24}$. Die Ergebnisse dieses Aufsatzes haben Eingang gefunden in die

${ }^{1} \mathrm{Cf}$ V. Haas, ChS I/1, $419 \mathrm{f}$

${ }^{22}$ Der 1987 erschienene Aufsatz (s. Anm. 12) wurde bereits 1984 im Manuskript abgeschlossen und zum Druck eingereicht.

${ }_{23}$ Neu, in: Festschrift für S. Alp (1992) 400.

${ }^{24}$ Wilhelm, Der hurritische Ablativ-Instrumentalis /net, ZA 73 (1983) 96-113. 
russische Überarbeitung von HuU durch M. L. Chačikjan ${ }^{25}$; der Aufsatz selbst gehört zu den bereits erwähnten, die von Diakonoff ins Russische übersetzt und publiziert wurden ${ }^{26}$.

Die formale Übereinstimmung der neuangesetzten Kasusendung mit dem sog. "Artikel“ -ne ist dem Verf. selbstverständlich nicht verborgen geblieben; einer herkömmlichen, aber unten in Frage gestellten Auffassung folgend, hielt er es zwar für möglich, die Formen auf $-n e / i$ mit ablativischer oder instrumentaler Bedeutung bei isoliert-morphologischer Betrachtung als Absolutive zu bestimmen $^{27}$, schrieb der Endung $-n e / i$ aber eine besondere Kasusfunktion zu, weil die Position des Absolutivs in einigen der angeführten Sätze bereits anderweitig besetzt war.

Ein neuer Aspekt des Sachverhalts ergab sich durch die 1983 gefundene hurritisch-hethitische Bilingue ${ }^{28}$. Hier tauchen Formen auf $-n e / i$ auf, die mit den Kategorien Ablativ und Instrumentalis nicht beschrieben werden können. Der Bearbeiter der Bilingue, E. Neu, bezeichnete 1988 diese Formen als „determinierte Stammformen . . . , die . . . auf die Frage 'Wohin?' stehen“29. Er bestimmt die fraglichen Formen also als Absolutiv und postuliert neben der Grundfunktion des Absolutivs (Kasus des Subjekts im nicht-ergativischen Satz und des Objekts im ergativischen Satz) eine weitere Funktion als Ortskasus.

Unter Beschränkung auf die vom Verf. zusammengestellten Belege aus dem Mit.-Brief erklären nun auch Dietrich und Mayer die fraglichen Formen auf $-n e / i$ als Absolutive ${ }^{30}$. Dabei weisen sie weder auf Neus im wesentlichen identische Interpretation der Formen auf $-n e / i$ in direktivischer Funktion noch auf die durch die Boğazköy-Funde vermehrte Materialgrundlage hin.

Der Sachverhalt selbst verdient eingehendere Erörterung. Wenn man wie Neu und jetzt auch Dietrich und Mayer die Formen auf -ne $i$ in dimensionaler Funktion als Absolutive interpretiert, so geht man davon aus, daß -ne/i nicht nur sprachgeschichtlich,

${ }^{25}$ Chačikjan, ChUJ 72, 74.

${ }^{26}$ Cf. oben Anm. 11

${ }^{27}$ Wilhelm, l.c. 101

28 Wilhelm, l.c. 101 .

${ }^{29}$ Neu, Das Hurritische, 32. Ausführlicher und mit breiterem Belegmaterial jetz Neu, Der hurritische Absolutiv als Ortskasus. Zur Syntax der hurritisch-hethitischen Bilingue aus Hattuša, in: Fs. Alp (1992) 391-400.

30 S. 110: „ein offensichtlich adverbial gebrauchter Absolutus oder 'Absolutus der Beziehung' ". sondern auch auf der Ebene des vorliegenden Sprachmaterials funktional identisch sei mit dem sog. „Artikel“ -ne und daß dieser bei jedem Kasus und also auch beim Absolutiv stehen könne. Eine solche Auffassung hat aber den Befund zumindest des MittaniBriefes - weitergehende Untersuchungen können im vorliegenden Rahmen nur exemplarisch vorgenommen werden - gegen sich. Der Mittani-Brief enthält keinen einzigen Absolutiv mit dem „Artikel“ -ne, läßt man einmal die hier zur Diskussion stehenden Formen auf -ne/i mit dimensionaler Funktion sowie unsichere Ergänzungen beiseite. Dabei spielt die Kategorie der Determination, auf die die Bezeichnung „Artikel“ Bezug nimmt, keine Rolle.

So steht zehnmal ${ }^{31}$ bezeugtem ōmini „Land" im Absolutiv Sg. ${ }^{32}$ keine einzige Form *ōminn=ne (Absolutiv mit „Artikel“ -ne) gegenüber, wohl aber dreimal der Genetiv $\bar{o} m \bar{i} n=n \bar{e}=v e^{33}$. Daß der "Artikel“ nicht unmittelbar determiniert, wird deutlich in Mit. II 69 und 71, wo zweimal - in beiden Fällen sinngemäß determiniert von „dem ägyptischen Land" die Rede ist, jedoch nur beim Genetiv, nicht beim Absolutiv der „Artikel“ Sg. steht.

Ohne daß an dieser Stelle die Diskussion um die Funktion des „Artikels" $\mathrm{Sg}$. in voller Breite wieder aufgegriffen werden könnte ${ }^{34}$, sei doch die Einschätzung geäußert, daß eine relationale gegenüber der determinierenden Funktion ${ }^{35}$ die wahrscheinlichere ist, zumal sie mit der relationalen Funktion von -ne bei der Suffixaufnahme besser harmoniert. Als weitere Beispiele seien angeführt: Fünfmal bezeugtes niḡâ/âri „Mitgift“ im Absolutiv Sg. ${ }^{36}$, jedoch kein *niğār=re, dagegen der Genetiv ni $\ddot{g} \bar{a} r=r \bar{e}=v e$ (Mit. iii 41) und der

${ }^{31}$ Bei den Zahlenangaben werden im folgenden nur gut erhaltene Belege berücksichtigt.

${ }^{32}$ Mit. i 20,90 , ii 68,69 , iii $6,7,24,113,118,120$.

${ }^{33}$ Mit. i 62 , ii $71,72$.

${ }^{34}$ Cf. F. Thureau-Dangin, Syria 12 (1931) 254-256; J. Friedrich, AnOr. 12 (1935) 127 f.; A. Goetze, RHA 39 (1940) 200 f.; Speiser, IH 98-102; Bush, GHL 148-154; A. Kammenhuber, MSS 23 (1968) 49-79; Diakonoff, HuU 87.

${ }^{35}$ Cf. u. a. jeweils ohne -ne: allai "die Herrin“ (des ägyptischen Landes) Mit. i 62 šăla "die Tochter" („meines Vaters" bzw. „Großvaters" bzw. „des mittanischen Herrn“) Mit, i 47, iii 35, 37, 104, paššst thi der Gesandte" (meines Bruders") Mit. ii 57 , iv 35 (cf. auch pašš ithe einen Gesandten“ Mit. iv 54), salamž $i$,die/eine Statue" Mit. iii 90, 97, 99, 102, 106

${ }_{36}$ Mit. ii 14 , iii $15,34,42$, iv 48 . 
Ablativ niḡâr $=r \bar{e}=d a n$ (Mit. ii 61). ${ }^{37}$ Zweimal taže "Geschenk" im Absolutiv Sg. (Mit. I 85, 90), jedoch kein *taže=ne, dagegen je zweimal der Genetiv und der Dativ $\operatorname{taz} \bar{e}=n \bar{e}=v e$ bzw. $=v a^{38}$. Zwölfmal tive "Wort ${ }^{* 39}$, jedoch kein *tive=ne, dagegen einmal der Dativ tive $=$ $n \bar{e}=v a$ Mit. i 110 und einmal der (verkürzte) Instrumentalis ${ }^{40}$ tive $=n e=$ $n$ Mit. iv 33

Eine umfassende Untersuchung am hurritischen Sprachgut außerhalb des Mit.-Briefes steht noch aus; wegen des häufg noch fehlenden lexikalischen und syntaktischen Verständnisses bleiben hier manche Ambiguitäten. Man vergleiche aber in der hurr.-heth. Bilingue nāli (Absol.) „Reh“ KBo. 32,14 Vs. i 9, 12 (bezogen auf das ibid. Z. 1 eingeführte und damit zweifellos determinierte Reh) gegenüber $\bar{e} r b i=n e=z ̌$ (Erg.) "Hund“ KBo. 32,14 Rs. IV 9 und kame $\bar{e}=$ $n e=\check{z}$ (Erg.) $\left(\right.$ Tier $\left.^{41}\right)$ Rs. 23 am Anfang eines Gleichnisses und damit indeterminiert ${ }^{42}$

Auch bei Namen ist dasselbe Phänomen zu beobachten: Der Name des Helden im Kešši-Lied erscheint als Kešše im Absolutiv

${ }^{37}$ Bei der Auswahl der Beispiele sind diejenigen Belege ausgesondert worden, die sicher oder wahrscheinlich das Possessivsuffix der 3. Ps. Sg. enthalten, da dieses mit dem "Artikel“ -ne inkompatibel ist.

${ }^{38}$ Mit. i 91, 92, 99, 104.

${ }^{39}$ Mit. i 83 , ii $12,79,101$, iii 49 , iv $2,4,13,14,17,19,107$.

40 Dietrich und Mayer, $113 \mathrm{f}$, scheinen die Instrumentalis-Funktion des Kasus auf -ne/i gänzlich leugnen zu wollen, ohne allerdings auf das Belegmaterial aus Boğazköy einzugehen. Thr auch sprachlich mißglückter Einwand (S. 114 oben) gegen die Auffassung von Mit. iv 32f. und EA 29:14 als Quasibilingue ist trivial, da grundsätzlich zu erwarten ist, daß strukturell verschiedene Sprachen einen gegebenen Sachverhalt unterschiedlich kodieren. Im vorliegenden Falle ist es nur von Interesse, daß sowohl in dem akkadischen als auch in dem hurritischen Satz der Sprecher bestretet, in Herz des Adressaten gekränkt zu haben, und dabei das instmumentum einer solchen möglichen Kränkung, nämlich irgendein Wort" bzw. „ein einziges Wort“, nennt. - Dietrich und Mayer weisen dem Kasus auf $-n e / i$ an dieser Stelle ohne weitere Begründung und ohne Parallelen eine lokativische Bedeutung zu (,in der Sache“). Sie ignorieren die von Ch. Girbal, ZA 78 (1988) 124 erkannte Bedeutung von šukkanne (hervorgehobenes „ein“), obwoh diese sich durch das von der hurritisch-hethitischen Bilingue gebotene Zahlwort šukki ,einmal“ (cf. Neu, Hethitica 9 [1988] 163, 170 Anm. 43; Or. 59 [1990] 232) glänzend bestätigt hat.

41 Cf N Oettinger, in: Der Äsop-Roman (1992) 11 mit Anm. 26

${ }^{42}$ Die Möglichkeit, daß hier die der Fabel gemäße generische Determination vorliegt, darf man mit Hinweis auf die anderen Gleichnisse ausschließen, in denen das handelnde Tier oder Objekt stets ohne -ne eingeführt wird, sofern es im Absolutiv steht: nā $l i$ „Reh“ KBo. 32,14 Vs. i 1, 26, kāai („Becher“) 42, [ku]mdi (,Turm“) Rs. 35, [t]āli „Baum“ Rs. 55.
(KBo. 12,79:5'; KUB 47,1 Vs. ii $4^{\prime \prime}$ ), jedoch als Kešše $=$ ne= im Ergativ KUB 47,2 Rs. iv 6, 10, Rd. 8 und im Genetiv(!) KUB 47,2 Vs. i 12. Ebenso der Name des Sonnengottes: Šimīge im Absolutiv Mit. iv 122, ChS I/1 41 Vs. i 50, Rs. iv 15, 16; I/5 30:3', Šimīge $=n \bar{e}=$ im Ergativ Mit. i 77, 106, iv 125; ChS I/1 5 Vs. i $53 ; \mathrm{I} / 570$ Vs. ii 10', 75 Vs. i 14', Genetiv Mit. i 86, 94; ChS I/1 9 Vs. i 54; I/2 79 Vs. i 6'; I/5 57 Rs. iii 15', Dativ Mit. i 87, 105; ChS I/1 5 Rs. iii 27 und Direktiv ChS I/1 5 Rs. iii 25.

Dasselbe Muster findet sich bei dem Namen Kumarbi; die Tatsache, daß es sich hierbei um einen "freischwebenden Genetiv handelt, hat also anscheinend keinen Einfluß auf die Verwendung des „Artikels": Kumarbi im Absolutiv ChS I/2 40 Vs. i 13 (Absolutiv als Vokativ), I/5 1 u. Rd. 24' (Text: -mar-), Kumarbi=ne= im Ergativ KBo. 12,80 Vs. i $14^{\prime}$; KUB 45,61 Vs. ii $8 ; 47,2$ Vs. i $11 ; 47,48$ Vs. 14', Genetiv ChS I/1 11 Vs. 31; I/5 89 Vs. ii 8, Direktiv ChS I/1 5 Rs. iii 11 und Komitativ KUB $45,62+$ Vs. i 13.

Die Auffassung, die Formen auf $-n e / i$ in direktivischer, ablativischer und instrumentaler Funktion seien als Absolutive zu betrachten, scheitert also daran, daß der Absolutiv mit dem Artikel" -ne nicht kompatibel zu sein scheint. Selbst wenn dies nicht in dieser Ausschließlichkeit zuträfe und vereinzelte Absolutive mit "Artikel“ -ne nachgewiesen werden könnten, bliebe ein Widerspruch zwischen der obligatorischen ${ }^{43}$ Anwesenheit von -ne/i bei den Formen in direktivischer, ablativischer und instrumentaler Funktion und dem regulären Fehlen des "Artikels“ -ne beim Absolutiv, wie es die hier zusammengestellten Formen belegen Wahrscheinlicher ist es, daß dem "Artikel“ oder besser relationalen Suffix -ne in den vom Verf. und Neu gesammelten Fällen eine allgemeine relationale Kasusfunktion zukommt, die teilweise durch die Entwicklung spezieller Kasus zurückgedrängt wird ${ }^{44}$. Dazu würde passen, daß der Kasus auf -ne im archaischeren Hurritischen von Boğazköy ebenso wie im (in dieser wie in anderer Hinsicht altertümlichen) Urartäischen deutlich häufiger vorkommt als im Mittani-Brief.

${ }^{43}$ In seiner Zusammenstellung der direktivischen Formen auf $-n e / i$ verweist Neu, in: Fs. Alp, 400, auf die Form negri, die er bereits früher (Das Hurritische, 25) als Absolutiv ohne -ne in lokativischer Bedeutung interpretiert hat. Für eine alternative Deutung, die die Annahme eines Sonderfalles zu vermeiden sucht, ef. Wilhelm, Or. 61 (1992) 132 f. sub negri.

44 Ähnlich V. Haas/I. Wegner, OLZ 86 (1991) 390. 


\subsection{Das pronominale Suffix $-n /-n n a$}

Seit den Anfängen der Erforschung des Hurritischen gibt es eine Diskussion um das Suffix $-n$. In der Pionierphase hielt man das Suffix für eine Akkusativendung; mit zunehmendem Verständnis der Struktur des Hurritischen wurde es zunächst als Kopula pronominaler Herkunft ${ }^{45}$, dann als Pronomen der 3. Ps. Sg. und Allomorph von -nna gedeutet ${ }^{46}$. Wesentliche Klärungen der Morphologie, Syntax und Funktion von $-n /-n n a$ hat W. Farber geliefert ${ }^{47}$, und einige Fälle, in denen der pronominale Bezug auf die 3. Ps. Sg. problematisch erscheint, hat Ch. Girbal gesammelt ${ }^{48}$. Wiederholt ist dabei die Frage diskutiert worden, ob das Suffix $-n$ am Jussiv mit diesem pronominalen Element identisch sei oder zumindest denselben Ursprung wie dieses habe.

Dietrich und Mayer liefern zu dieser Diskussion einen Beitrag, den man in doppeltem Sinne originell nennen möchte. Sie verzichten - nicht ohne Pauschalkritik an ihren Forschungsvorgängern ${ }^{49}$ - auf eine Diskussion der komplizierten Fragen, die sich aus der offenkundigen funktionellen Diversität und der wahrscheinlichen etymologischen Identität zahlreicher $n$-haltiger Endungen des Hurritischen ergeben. Die beiden Autoren schlagen nun eine radikal neue Lösung vor, nämlich die Aufgabe der Endung $-n$ als eines morphologischen Phänomens überhaupt. Threr Meinung nach ist $-n$ nichts anderes als der graphische „Nie derschlag einer Nasalierung des Auslautvokals" (S. 110). Sie stellen dabei eine Verteilungsregel auf, die auf Grund sprachlicher Unzulänglichkeit, stehengebliebener Druckfehler und der um mehrere Argumentationsschritte verkürzten Darstellung unverständlich bleibt ${ }^{50}$. Anscheinend ist hier nicht mehr (oder nicht nur?) wie zuvor von dem enklitischen Pronomen - $n /-n n a$ und dem $-n$ beim Jussiv die Rede, sondern (vor allem?) von dem bisher als

${ }^{45}$ Speiser, IH § 208

16 A Goetze, JCS 2 (1948) 259-261.

7 Farber, Or. 40, 29-66.

${ }^{4}$ Girbal, ZA 80, 93-101.

${ }^{49}$ S. 109 zu den Anm. 47 und 48 genannten Arbeiten von Farber und Girbal: „ohne neue Ergebnisse“; „Wir haben nicht die Absicht, uns mit diesen Beiträgen auseinanderzusetzen."

50 UF 23, 110 oben: Betrachtet man alle Belege für $-n$, dann ergibt sich, daß die enklitischen Partikel(sic!) und die abhängigen PersPron. im absoluten Wortauslaut immer dieses haben, während es bei letzterem(sic!) im Inlaut, also vor enklitischen Partikeln, nie erscheint. enklitisches Konnektivum gedeuteten $-a n^{51}$, auf das aber mit keinem Wort Bezug genommen wird. Es bleibt abzuwarten, ob die beiden Autoren sich in der Fortsetzung ihrer geplanten Aufsatzserie als fähig erweisen, ihre Interpretation des Sachverhalts angemessen zu beschreiben und so einer Beurteilung zugänglich zu machen $^{52}$

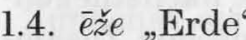

In den kaluti-Listen aus Boğazköy entspricht das Wortpaar ēže havurmi sumerographischem AN KI, akkadographischem $\check{S} A-M E-E E R-$ SE-TIM und heth. nepiš tekan/daganzipaš, jeweils „Himmel (und) Erde“. Dies führte C.-G. von Brandenstein 1937 zu dem Schluß, ēze entspreche „Himmel“ und havurmi „Erde" ${ }^{\text {"53 }}$. Seine Deutung wurde allgemein akzeptiert ${ }^{54}$, bis durch die 1983 gefundene hurritischhethitische Bilingue klar wurde, daß die Wortfolge im Hurritischen nicht der der anderen altvorderasiatischen Sprachen entspricht, sondern umzukehren ist: ēže ist „Erde“, havurmi „Himmel“. Die Diskussion der entscheidenden Textstellen KBo. 32,11 i $3 / / 13$ i 12 f. und KBo. 14 Rs. 35 f. (cf. noch 57), die Neu während des 2. Konstanzer Altorientalischen Symposions 1986 bekanntgab $^{55}$, erbrachte das Ergebnis, daß keine Stelle der hurritischen Literatur gegen eine solche Umkehrung der Bedeutungen spricht, daß im Gegenteil ein von E. Laroche hinsichtlich der Semantik emendierter Listeneintrag mit der Gleichung hurr. [ha-]bur-ni = ugarit. $[\xi] a-[m] u-{ }^{\top} m a^{156}$ als Bestätigung gewertet werden darf ${ }^{57}$. Bibliographisch faßbar ist die neue Erkenntnis (mit Nennung Neus) erstmals in der französischen Übersetzung des Mittani-Briefes, die Verf. $1987 \mathrm{zu}$ der Gesamtïbersetzung der Amarna-Briefe durch W. L. Moran beigesteuert hat ${ }^{58}$.

51 Friedrich, MVAeG 42/2 (1939) 14-22; Speiser, IH § 211; Bush, GHL 262-268; Diakonoff, $\mathrm{HuU} 150 \mathrm{f}$

${ }^{52}$ Nur am Rande sei erwähnt, daß S. 109 unten der transitive Jussiv der 3. Ps. Sg. $=i=e n)$ fälschlich als $=e n$ angesetzt und außerdem mit dem transitiven Imperativ 2. Ps. Sg. verwechselt wird.

${ }^{53}$ C.-G. von Brandenstein, ZDMG 91 (1937) 571 Anm. 1; ZA 46 (1940) 85-89.

${ }^{54}$ Cf. u. a. Friedrich, MVAeG 42/2, 30; Speiser, IH S. 74; Bush, GHL 104, 335 Anm. 93; Laroche, GLH 83 f.; Diakonoff, HuU 60.

${ }_{55}$ Cf. Neu, in: Haas (Hrg.), Hurriter und Hurritisch (Xenia 21, 1988) 114 Anm. 12 ders., Das Hurritische (1988) 15 Anm. 32, $26 \mathrm{f}$.

${ }^{56}$ Laroche, UF 11 (1979) 477-480.

57 Cf. Wilhelm apud Neu, Das Hurritische (1988) 26 f. Anm. 80

58 W. L. Moran, Les Lettres d'El Amarna (1987) 151. 
Wenn Dietrich und Mayer S. 111 diesen forschungsgeschichtlichen Sachverhalt mit der Formulierung verunklaren, „die Bedeutung von $\bar{e} z ̌ e=$ wurde inzwischen . . . auch von G. Wilhelm in 'Erde' geändert“, und dies als „cum grano salis richtiges Ergebnis“ qualifizieren, so mag man dies als Besserwisserei ex post abtun. Es kann aber nicht hingenommen werden, daß die beiden Autoren der bisherigen Forschung ${ }^{59}$ den Vorwurf machen, „das nächstliegende(sic!) übersehen" zu haben, nämlich den etymologischen Zusammenhang von hurr. èze "Erde" und urart. esi „Ort, Platz"60. Es gehört zu den methodologischen Elementarforderungen des wissenschaftlichen Sprachvergleichs, nur solche Wörter zu vergleichen, deren Semantik in der jeweiligen Einzelsprache auf kombinatorischem Wege unabhängig voneinander ermittelt wurde ${ }^{61}$. Wer vor Kenntnis der Bilingue und damit vor dem kombinatorischen Nachweis der Bedeutung „Erde“ eine solche Bedeutung nur auf Grund von urart. esi angesetzt hätte, hätte gegen die heuristische Methodik verstoßen.

Wenn Dietrich und Mayer nun ohne Diskussion der Kontexte die Bedeutung von urart. esi auf alle Belege für ēže im Mit.-Brief anwenden wollen (S. 111), so ist dies ein weiterer Verstoß derselben Art. Daß ein solcher Bedeutungsansatz keinesfalls überal möglich ist, zeigt schon die Wendung e-e-še-né-e-ra ha $a$-a-wu-ruun'-né-e-ra Mit. iii $100 \mathrm{f}$., wo éžze syntaktisch parallel mit havurn „Himmel“ steht und damit ebenso wie in den Listen aus Boğazköy und in der Bilingue „Erde“ bedeutet.

Die oben angeführten Beispiele mögen genügen, um zu zeigen, daß die Kritik von Dietrich und Mayer an den Ergebnissen der neueren hurritologischen Forschung einer hinlänglichen Begründung entbehrt und in mindestens einem Fall auf einem ausgesprochen gewaltsamen Umgang mit den Quellen basiert. Um so unerfreulicher ist der dabei durchweg anzutreffende belligerente

59 Cf. Anm. 53 und 54; die beiden Autoren versuchen allerdings den Eindruck zu erwecken, es handele sich hier um ein Versäumnis, das besonders dem Verf. anzulasten sei.

60 Denselben Zusammenhang hat Girbal unabhängig davon bereits in einem Anfang 1989 zum Druck eingereichten Beitrag zu der bis heute (März 1993) nich erschienenen, von Y. L. Arbeitman geplanten Gedenkschrift für Ch. Carter hergestellt.

61 In dest altkleinasiatischen Philologie hat vor allem J. Friedrich immer wieder auf dientringen; cf. z. B. MVAeG 42/2, 62 .
Ton, der die Einsicht vermissen läßt, daß Wissenschaft ein Diskurs, kein Feldzug ist.

2.

Im folgenden sollen einige der lexikalischen und grammatikalischen Eigenbeiträge der beiden Autoren kommentiert werden. Zunächst sei jedoch ihre Umschriftweise ${ }^{62}$ kurz charakterisiert: Dietrich und Mayer geben in der syllabischen Umschrift das Zeichen GI teils als gi (S. 111, Mit. iv 125, S 112 Z. $86 \mathrm{f}$.), teils als $g e$ S. 112 Mit. i 83) wieder, ohne die wohlbekannte Regel zu beachten, daß im Mit.-Brief (und weithin auch in Nuzi) GI einen Tektal mit eVokal und KI einen Tektal mit $i$-Vokal bezeichnet. Der Name des Sonnengottes lautet deshalb bei ihnen fälschlich "Šimīgi“ statt richtig Šimige. In der gebundenen Umschrift setzen sie diejenigen Konsonanten ein, die sich aus dem häufigsten Lautwert des jeweils verwendeten Keilschriftzeichens ergeben. Die dafür gebotene Begründung, der Mit.-Brief unterscheide Mediae und Tenues „im Rahmen der Möglichkeiten der Keilschrift sorgfältig und konsequent" (S. 109), ist nicht stichhaltig. Das Zeicheninventar des Mit. Briefs ist, wie zuletzt F. W. Bush ausführlich dargestellt hat ${ }^{63}$, höchst ökonomisch und trifft bei den Zeichenpaaren, die eine Sonoritätsopposition darstellen, meist eine Auswahl. So werden stets PA, TA, KA, TE, TI und DU, nie BA, DA, GA, DI und TU verwendet. Dies allein zeigt, daß die Orthographie des Mit.-Briefes keineswegs die zur Zeit der Niederschrift des Textes bestehenden Möglichkeiten zur Darstellung der Sonoritätsopposition einsetzt. Eine Wiedergabe der Stimmhaftigkeit oder Stimmlosigkeit eines Konsonanten war für den hurritischen Schreiber ebensowenig nötig, wie für die deutsche Orthographie eine Unterscheidung der (phonetisch verschiedenen) Frikative in „echt" und „acht" erforderlich ist; denn in beiden Fällen handelt es sich um Allophonie. Die Allophonieregeln, nach denen sich die Stimmhaftigkeit von Konsonanten bestimmt, sind seit langem bekannt und brauchen hier nicht wiederholt zu werden. Sie sind keineswegs nur aus den alphabetischen Texten aus Ugarit abgeleitet worden, wie Dietrich

${ }^{62}$ Die Transliteration der Textstellen des Mit.-Briefes ist nicht immer verläßlich; S. 112 Mit. i 83 lies $p a-a \check{s}-s ̌ i-i-\ldots$, i 86 lies -ma-a-an, S. 114 Mit. ii 17 lies $p u-u k$ lu-ša-a-un-. . , ii 87 lies: tup-pu-u-un-ni. IB wird als $i w$, nicht als $i w$ wiedergegeben, obwohl $i w$ nach Akk.Syll. Nr. 223 eine Lesung des Zeichens PI ist.

63 Bush, GHL 18-22. 
und Mayer annehmen (S. 109), sondern unabhängig davon auch aus der Niederschrift hurritischer Personennamen durch babylonische Schreiber in Nuzi, Nippur, etc. ${ }^{64}$ Ein völliges Mißverständnis dieses Sachverhalts liegt vor, wenn die beiden Autoren die von Speiser begründete und von Diakonoff und anderen, darunter dem Verf., konsequent angewandte Notierung der Konsonantenallophone nach den bekannten Distributionsregeln als „heute üblichen Brauch, die Mediae durch Tenues und umgekehrt wiederzugeben", beschreiben (S. 109).

Einen Rückschritt hinter die Erkenntnis, daß im Mit.-Brief die Zeichen U und Ú eine Phonemopposition wiedergeben ${ }^{65}$, stellt die bei Dietrich und Mayer anzutreffende undifferenzierte Transkription der beiden Zeichen als - $u$ - dar. Der Ansatz eines Vokalsystems mit fünf Vokalqualitäten wird in aller wünschenswerten Klarheit durch die Schultafel Msk. 7462 aus $\operatorname{Emar}^{66}$ bestätigt:

$$
\begin{aligned}
& \text { WA- } u: \mathrm{BU}-u \\
& \text { WA- } a: \mathrm{PA}-a \\
& \text { WA- } e: \mathrm{BE}-e \\
& \text { WA- } i: \mathrm{BI}-i \\
& \text { WA- } u \text { : } \mathrm{BU}-u
\end{aligned}
$$

Erfreulicherweise folgen die beiden Autoren der vom Verf. einge führten und jüngst begründeten ${ }^{67}$ Praxis, plene geschriebene Vokale in der gebundenen Umschrift zu kennzeichnen; es sei ihnen nachgesehen, daß sie sich dabei nicht auf den Verf. berufen, der hierin bisher allein stand, sondern fälschlich auf ein „heute gängiges Muster“ (S. 109).

In grammatikalischer Hinsicht ist die auffälligste Neuerung, die Dietrich und Mayer vorschlagen, die Einführung des Begriffs der „possessiv-pronominalen Deklination“ (S. 109, 121), der von Diakonoff für das Urartäische geprägt wurde, da dort „die einzelnen Suffixpositionen nicht mehr so klar abzusondern"68 seien. Eine Begründung, warum die Possessivsuffixe und die Kasusendungen im Hurritischen nicht klar zu trennen sein sollen,

${ }^{64}$ Cf. P. M. Purves, AJSL 57 (1940) 172-185.

65 Dasselbe gilt bekanntlich für KU und GU, die konsequent für Tektal mit $o$-Vokal bzw. Tektal mit $u$-Vokal verwendet werden.

66 Jf. . bu-ú!).

67 Wilhelm, Or. 61 (1992) 125 (mit einer wichtigen Präzisierung Z. 2 f.!)

68 Diakonoff, HuU 89 liefern die Autoren nicht. Ein Blick in die geläufigen Grammatiken des Hurritischen (mit einer geringfügigen Präzisierung beim Possessivsuffix der 1. Ps. Sg. ${ }^{69}$ ) lehrt, daß das Hurritische leicht segmentierbare und strikt monofunktionale Nominalsuffixe kennt. In keinem Falle sind pronominale Funktionen mit Kasusfunktionen in einem einzigen Grammem kombiniert. Der Begriff einer „possessiv-pronominalen Deklination“ hat also keinen Platz in der hurritischen Grammatik.

Ein weiterer Versuch, die hurritische Grammatik zu ergänzen, hält ebenso wenig einer Nachprüfung stand. Dietrich und Mayer glauben ein Possessivsuffix der 2. Ps. Pl. $-a p / w$ entdeckt zu haben, das dem von ihnen als $-i p / w$ angesetzten Possessivsuffix der 2. Ps. Sg. gegenüberstehe ${ }^{70}$. Der Vokal, der die angebliche Differenzierung ausmacht, gehört indes nicht zum Possessivsuffix, sondern zum vorausgehenden Substantiv ${ }^{71}$. šāla „Tochter" und šèna „Bruder" sind bekanntlich wie andere Verwandtschaftsbezeichnungen $a$-Stämme. Es besteht also kein Grund, den Ansatz des Possessivpronomens der 2. Ps. Sg. zu revidieren; es lautet im Mit.-Brief - $v$ - vor $-u$-, $-b$ - vor $-a$ - und - $p$ - vor Konsonant; die Schreibungen im Auslaut sind ambivalent $(-v / b)$. Das Possessivsuffix der 2. Ps. Pl. ist - was den beiden Autoren anscheinend unbekannt geblieben ist - KBo. 32,19 Vs. i 15, 16 und 23 (u-ul-mi-iš-ši „,eure Waffen“, $e-t e-s ̌ u-u ́-t a$ „zu eurem Körper“) bezeugt und enthält, wie auf Grund der Possessivpronomina der 1. und der 3. Ps. Pl. (iff=až,

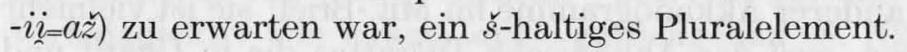

${ }^{69}$ Cf. Wilhelm, Or. 54 (1985) 488, Z. $10 \mathrm{ff}$.

${ }^{70}$ Wenn die beiden Autoren den entsprechenden Absatz ihres Aufsatzes (S. 121) mit der Bemerkung einleiten: „In der Behandlung der possessiv-pronominalen Deklination der 2. Person herrscht in G. Wilhelm, Lettres, Unklarheit", so wird hier einmal mehr deutlich, in welchem Maße die beiden Autoren ad personam und nicht ad rem argumentieren. Verf. hat in der genannten Arbeit die Formen mit dem Possessivsuffix der 2. Ps. nicht anders übersetzt, als dies der communis opinio von den Anfängen der Hurritologie bis heute entspricht.

11 In den von Dietrich und Mayer als Dativformen gebuchten Belegen Mit. ii 114 und iv 14 liegen die Verbalwurzeln $t \bar{a} n$ - „machen“ und kad- „sprechen“ mit der Wurzelerweiterung -upp- vor; zu dieser cf. Speiser, IH 138, und Bush, GHL 180, sowie Laroche, PRU 3, 317, und Bush, GHL 173 zu elam=u/ol(l)=upp-. Der Beleg für den Lokativ ist im Lexem frei ergänzt (Text: $[\ldots-m] a-a-p a)$. 


\section{3.}

Bei der folgenden Diskussion einiger Beiträge der beiden Autoren zum hurritischen Lexikon werden nur solche Wörter berücksichtigt, die sie in ihrem Kommentar besprechen, nicht dagegen solche, für die nur in den Textübersetzungen neue oder modifizierte Bedeutungen geboten werden.

\section{1. salamži "Statue"}

Mehrfach drückt der Absender des Mit.-Briefes seinen Wunsch nach Übersendung einer Statue aus, die seine an den Pharao verheiratete Tochter Tatuhepa (Tādohēeba) darstellen soll. Das entsprechende hurritische Wort - salamž $i$ - wurde bereits unmittelbar nach der Veröffentlichung des Textes von P. Jensen richtig gedeutet und von akkad. șalmu "Statue" abgeleitet ${ }^{72}$. E. A. Speiser isolierte in salamži das Suffix -(a)šše, $-z ̌ i$, dem er eine Abstrakta bildende Bedeutung zuschrieb ${ }^{73}$.

Dietrich und Mayer deuten nun (S. 109) salamži als Akkadogramm mit dem akkadischen Possessivsuffix -ši, das sich jeweils auf Tatuhepa beziehe („ihr Standbild“, S. 109). Die beiden Autoren wollen damit die Annahme einer "Sonderbildung *zalamši < șalmašše vermeiden.

Eine solche Deutung ist nicht nur unwahrscheinlich angesichts des Fehlens anderer Akkadogramme im Mit.-Brief; sie ist vielmehr ausgeschlossen, weil das Akkadische Tušrattas die in Ugarit und vor allem Emar übliche Verwendung von $-s ̌ i$ als Possessivsuffix der 3. Ps. f. Sg. nicht kennt, vielmehr sorgfältig zwischen dem Possessivsuffix am Nomen $-s ̌ a$ und dem Personalsuffix des Akkusativs am Verb $-s ̌ i$ unterscheidet ${ }^{74}$

Gegen die Deutung von Dietrich und Mayer spricht weiterhin, daß salamž $i$ keineswegs eine „Sonderbildung“ ist. Zwar ist die von Speiser als Parallele gebotene Form *tamgar $z i^{75}$ logographisch als DAM.GÀR-š $i \mathrm{zu}$ verstehen und nach syllabischen Schreibungen tamgarašše $/ i$ zu lesen ${ }^{76}$. Es gibt aber mehrere hurritische Wörter, deren Basislexem auf $-m,-n$ oder $-r$ auslautet und die alle das Allo-

${ }^{72}$ P. Jensen, ZA 5 (1890) 189

73 Speiser, IH § 163.

74 Cf. H.P. Adler, AOAT 201 (1976) 323, 329 s.v.

${ }_{75}$ Speiser, IH $\S 163$.

${ }^{76}$ Cf. C. Zaceagnini, Iraq 39 (1977) 186 ff. (Bedeutung: „Gewinn“). morph -ži aufweisen. Darüber hinaus findet sich die in salamž $i$ zu beobachtende Anaptyxe nach Art der akkadischen status constructus-Bildung von $p$ Vrs auch sonst: hurr. $t i b i s ̌=s ̌ i$ "Strohschober" geht - wohl über hurr. *tibni - auf akkad. tibnu „Stroh“ zurück. Daß diese Anaptyxe aber innerhurritisch erklärt werden kann, zeigt hurr. tumuššs $i$,vierter", das von tumni „vier" abgeleitet ist. Es kann auch nicht als Argument zugunsten der Auffassung von Dietrich und Mayer gelten, daß das Konkretum salamži eine Endung trägt, die Abstrakta bildet; denn mit dieser Funktion ist die Endung nicht hinlänglich beschrieben, wie verschiedene Gebäudebezeichnungen zeigen (pidarži „Rinderstall“ zu pidari "Rind“77, tibišš ${ }^{\prime} i$ "Strohschober" [s.o.], dazu wohl auch trotz $l$ Anlauts É(. . .) li-ip-pu-ur-šu HSS 19,5:5, 17; 47:6, 16). Die vom Verf. bei früherer Gelegenheit gebotenen Beispiele ${ }^{78}$ lassen sich noch vermehren: *puramži „Sklaverei“79 zu purame „Sklave“ sowie die Opfertermini aus Boğazköy kamerž $i^{80}$ und niğarži $i^{81}$.

$$
\text { 3.2. abli, padi, kimr-, àvattu-, šim- }
$$

Dietrich und Mayer liefern mehrere neue Bedeutungsvorschläge, die aus den bereits oben angesprochenen Gründen der Methodik nicht akzeptiert werden können, da sie nicht nach der kombinatorischen Methode, sondern durch Heranziehung des akkadischen Wörterbuchs gewonnen worden sind. So soll das hapax legomenon abli (Attribut von taže „Geschenk") Mit. i 85 auf das (unbezeugte) Verbaladjektiv von akkad. apālu zurückgehen (S. 112). Das ungedeutete Wort padi (Mit. iv 33, 67, 68, 72) wird mit assyr. battu(m) „Seite“ erklärt (S. 113), das unklare hapax legomenon ki-im-ra-aMit. ii 87 wird mit akkad. gimm "Gesamtheit" in Zusammenhang gebracht (S. 115); das ebenfalls aus dem Kontext heraus nicht bestimmbare $a-a-w a-a d-d u$-tan Mit. ii 11, $a-a-w a-a d-d u h-h a$ Mit. iii 17 wird von akkad. awatu „Wort, Sache“ abgeleitet (S. 118). Selbst eine akkadische Verbalwurzel wird zur Erklärung herangezogen: Die Wurzel šim- in der morphologisch und semantisch unklaren Form ši-mì (LUM)-i-ki Mit. iii 46 soll mit akkad. šiāmu „festsetzen,

\footnotetext{
Cf. Wilhelm, OrAnt. 22 (1983) 312

78 Wilhelm, ZA 78 (1988) 282.

${ }^{79}$ Erschlossen aus pu-ra-am-š (nach dem Kontext „Sklaverei“) HSS 19,42:3.

${ }^{80}$ Cf. Haas/Wilhelm, AOAT-S 3 (1974) 85

${ }^{81}$ Cf. Haas/Wilhelm, l.c., 111; CHD L-N 425 a.
} 
bestimmen" identisch sein ${ }^{82}$. Der Leser, dem die Geschichte der Hurritologie gegenwärtig ist, fühlt sich an eine Einschätzung der Forschungen Ferdinand Borks durch Johannes Friedrich erinnert: „Wo aber die kombinatorische Methode nicht weiterhilft (und das ist bei dem nicht unerschöpflichen Sprachstoff unseres Briefes nur zu oft der Fall), gehört Bork nicht wie der Verfasser zu jenen 'vorsichtig sein Wollenden', 'die in das zertrümmerte Mosaikbild hie und da ein Steinchen einpassen ${ }^{\prime 83}$. . A A Aer seiner Phantasie hat Bork noch ein anderes Mittel zur Deutung dunkler Wörter, die etymologische Methode, den Anklang an ein Wort einer bekannten Sprache ... Am meisten müssen angebliche Lehnwörter aus dem Akkadis chen ... herhalten, um für ungedeutete Wörter des Mitannibriefes eine Deutung abzugeben." 84

Es steht zu hoffen, daß die Wiederbelebung der von Friedrich kritisierten "Methode" keine Schule macht und nicht neuerlich Diskussionen nötig werden, die die Hurritologie bereits vor mehr als einem halben Jahrhundert abschließend geführt zu haben meinte.

${ }^{82}$ Angesichts ihrer Bedenkenlosigkeit in der Zurückführung hurritischer Wörter auf akkadische verwundert es, bei Dietrich und Mayer S. 119 Anm. 51 lesen zu müssen, „völlig abwegig“ sei „der Versuch, eine Partikel $m \bar{a}$ „folgendermaßen

zu isolieren und diese dann auch noch mit dem mittel- und neuassyrischen $m \bar{a}$ zu verbinden". Immerhin ist der Ansatz von hurr. $m \bar{a}$,folgendermaßen" strikt znn gewonnen (cf. Verf., SMEA 24 [1984] 220 Anm. 10), auf die die beiden Kritiker indes nicht weiter eingehen.

Die Tatsache, daß auf die Partikel $m \bar{a}$ das enklitische Personalpronomen $-n(n a)$ und das enklitische Konnektivum -an folgen, halten Dietrich und Mayer für ein Gegenargument, obwohl es doch gerade diese Enklitika sind, die ganz regelmäßig an die Partikeln im Sinne der hurr. Grammatik antreten; ef. Bush, GHL 238 (im Anschluß an Speiser, IH § 126): „the term 'particle' refers to that class of Hurrian words ... which cannot append any of the nominal or verbal suffixes, but can only append one or more associatives" (unter 'associatives' versteht but can only append one or more associatives" (unter 'associatives' versteht
Bush, Speiser folgend, vor allem die enklitischen Personalpronomina, die Konnektiva -an, und $-m \bar{a} n$ sowie einige weitere Enklitika). Da Dietrich un Mayer nichts anführen, was gegen den Ansatz hurr. $m \bar{a}$ „folgendermaßen spricht, besteht keine Veranlassung, auf Grund ihres Widerspruchs von diesem Ansatz abzurücken. Für die Möglichkeit einer alternativen Interpretation, die richen Zusammentan lichen Zusammen und assyr. $m \bar{a}$,folgendermaßen" betrifft, glaubt Verf. trotz der unterschiedlichen Position im Satz an der Formulierung festhalten zu dürfen: „Die Übereinstimmung ... ist kaum zufällig."

${ }^{83}$ Zitat aus F. Bork, Der Mitanibrief und seine Sprache (Altkaukasische Studien I, Königsberg 1939) S. II.

${ }^{84}$ J. Friedrich, WZKM 46 (1939) $200 \mathrm{ff}$.

\section{Die Ikonographie des Mondgottes in der Glyptik des III. Jahrtausends v. Chr.*}

\author{
von E. A. Braun-Holzinger - Heidelberg
}

Der Mondgott spielt wie auch der Sonnengott im mesopotaischen Pantheon eine große Rolle. Während der Sonnengott jedoch beliebtes Bildthema war, läßt sich der Mondgott auf Darstellungen nur sehr selten nachweisen. Bisher wurde meist nur eine Gottheit, deren Hörnermütze von einer Mondsichel bekrönt wird, als Mondgott identifiziert. Berücksichtigt man jedoch auch den Aspekt des Mondgottes als Strahlengott, als Stadtgott von Ur, eventuell auch als Vegetationsgottheit und bezieht man die Siegellegenden in eine Untersuchung mit ein, lassen sich doch wesentlich mehr Götterdarstellungen mit dem Mondgott in Zusammenhang bringen.

Während sich Philologen eingehend mit Mythen und Kult des Mondgottes befaßten ${ }^{1}$, beschränkte sich die Beschäftigung der Archäologen mit diesem bedeutenden Gott des mesopotamischen Pantheons auf sparsame Hinweise, obwohl die Darstellungen auf der Stele des Ur-Nammu und auf einer Wandmalerei des Palastes von Mari deutlich machen, daß er im Bildrepertoire durchaus eine

\footnotetext{
Sonderabkürzungen: Boehmer, EGAZ = R. M. Boehmer, Die Entwicklung der Glyptik während der Akkad-Zeit. UAVA 4 (1965). - Buchanan, Ashmolean = B. Buchanan, Catalogue of Ancient Near Eastern Seals in the Ashmolean Museum (1966). - Buchanan, Yale = B. Buchanan, Early Near Eastern Seals in the Yale Babylonian Collection (1981). - Collon, WACS = D. Collon, Catalogue of the Western Asiatic Seals in the British Museum. Cylinder Seal II (1982); 1 (1986). - Delaporte, CCO = L. Delaporte, Musce du Loure. Catalogu cylindres, cachets et pierres gravées de style oriental I (1920); II (1923). - Frankfort, OIP $72=$ H. Frankfort, Stratified Cylinder Seals from the Diyala Region. OIP 72 (1955). - al-Gailani Werr, Bibl. Mes. $23=$ L. al-Gailani Werr, Studies in the Chronology and Regional Style of Old Babylonian Cylinder Seals. Bibl. Mes. 23 (1988). - Hall, Nanna/Suen = M. G. Hall, A Study of the Sumerian Moon-God Nanna/Suen (Ph. D. diss. 1985 Philadelphia [1988]) - Legrain, PBS $14=\mathrm{L}$ Legrain, The Culture of the Babylonians from their Seals in the Collection of the Museum. PBS 14 (1925). - Moortgat, VR = A. Moortgat, Vorderasiatische Rollsiegel (1940). - Orthmann, PKG 14=W. Orthmann, Der Alte Orient. Propyläen Kunstgeschichte 14 (1975). - Porada, CANES = E. Porada, Corpus of Ancient Near Eastern Seals in North American Collections I (1948)

1 Zuletzt ausführlich Hall, Nanna/Suen.
}

Zeitschr. f. Assyriologie Bd. 83, S. 119-135

(C) Walter de Gruyter 1993

ISSN 0084-5299 